\title{
Dirichlet Model: Its Consistency and Tracking Pattern Using Buying Behavior Data
}

\author{
Maradesa Adeleke ${ }^{1 *}$ and Akomolafe Abayomi Ayodele ${ }^{2}$
}

Department of Statistics, Federal University of Technology, Akure,Nigeria

*Corresponding Author Email: maradprime1@gmail.com

(iD) https://orcid.org/0000-0002-2692-4290

Received: $3^{\text {rd }}$ June, 2017/ Revised: $14^{\text {th }}$ November, 2017/ Accepted: $12^{\text {th }}$ December, 2017

CIAppStat-SL2017

\begin{abstract}
Dirichlet model is a standard choice for modeling customers' satisfaction and their preference which make the modeling of their buying behavior possible. In tracking consumers' behavior pattern with respect to 7 up Bottling Company Nig. Plc flavor, Dirichlet model provides theoretical benchmark for price promotions, advertisement, branding as well as been used to decide future purchasing pattern of consumers when it comes to the volume of Pepsi, Mirinda and 7up that they will purchase. This research was carried out based on consistency of Dirichlet model in tracking and forecasting purchasing pattern of customers. This was established through the evidence from variance-covariance matrix, probability vector and parameter vector, stochastic matrix and the probability of sale at the next proposed eight periods for the three flavors. The precision parameter and Bayes factor were used to test the goodness of fit of Dirichlet model so that the estimate of Dirichlet model can be relied upon. The distribution has been fitted to real life datasets for illustrating its practical behavior; based on the evidences from probability of sale at different proposed sales periods, Dirichlet model predicted that the probability of purchase is not stable for one product, but keeps varying from period to period. Only the Mirinda flavor has more stable sales throughout the proposed period with average0.569478 (56.9478\%), while Pepsi and 7up have low purchasing probability with average 0.368518 (36.85\%) and 0.361292(36.13\%), respectively. Therefore, it can be relied on the Dirichlet model for predicting
\end{abstract}


customers buying behavior pattern thereby enhancing efficient and consistent planning and decision making.

Keywords: Stochastic Matrix,Dirichlet model, Variance-covariance matrix, Correlation Matrix, Bayes Factor.

\section{Introduction}

\subsection{Introduction to research problem}

The Dirichlet Model was developed by Goodhardt, Ehrenberg and Chatfield (Ehrenberg 1959; Goodhardt, Ehrenberg \& Chatfield 1984). It is a model for the patterns of repeat purchases of the brands within a product category. It models simultaneously the counts of the number of purchases of each brand, over a period of time. It has been shown to be applicable to many product categories and to have substantial uses, particularly with regards to the analysis of what are known as the brand performance measures (Ehrenberg 1988; Ehrenberg \&Uncles 1999; Ehrenberg, Uncles \& Goodhardt 2003; Uncles, Ehrenberg \& Hammond 1995).

Dirichlet Modeling usually involves applying the model to generate estimates of the brand performance measures including market share, average purchase rate, penetration, purchase frequency, share of category requirements, $100 \%$ loyal, average portfolio size and repeat rate. These estimates are known as the ë theoretical and are compared to the ë observed which are calculated directly from the data (Ehrenberg, Uncles \& Goodhardt 2003). The procedures for calculating the theoretical valuesfrom the parameters of the Dirichlet Model traditionally have been considered to be fairy complex, which is true (Ehrenberg, Uncles \& Goodhardt 2003) and are not well documented. Consequently, the Dirichlet Model is usually applied through packaged software (Hewitt 1990; Kearns 2000; Uncles 1989). However, as the use and development of the model expand, there is a requirement to document the functional forms, and their derivations, for calculating the brand performance measures from the parameters of the Dirichlet Model. The Dirichlet Model is a probability density function. It specifies the distribution of purchases by a population of shoppers of each of the brands within a product category over a specified period of time. The category might be, for example, detergents, the period of time a year, the population of households (called shoppers) in Nigeria, and the set of about twenty brands of detergents available in supermarkets in that country. The data in this example 
would record for each household in the sample the purchases of each of the brands over the specified year. The Dirichlet Model is the combination of two probability density functions, the negative binomial distribution (NBD) and the Dirichlet multinomial distribution (DMD). In the Dirichlet Model the category purchase rate is assumed to have a NBD over the population of shoppers. The NBD is derived through specifying that each shopper's purchases for the category follow a Poisson process based on a category propensity. Over the population of shoppers these propensities have a gamma distribution. The purchases of the individual brands are assumed to have a DMD which is conditional on the category purchase rate. The DMD is derived through specifying that each shopper's purchases of the brands. It is on this note that tracking the purchasing pattern of consumers will be beneficial to researchers, entrepreneurs, decision makers and other policy makers regarding building a stable economy. This research work was based on evaluation of consistent performance of Dirichlet model in predicting the behavior of consumers' and the prospective consumers or customers with respect to their future purchasing pattern towards beverage such as Pepsi, 7 up and Mirinda.

\subsection{Research Problem}

Consumer behavior analysis serves as a backbone of competitors' and SWOT analysis (Strengths, Weakness, and Opportunity available to potential competitors and Threats) which provide solid basis for developing frame work in order to build an effective marketing strategy through evaluation of public acceptance of their product by investigating the consumption habit of the populace which constitutes their customers. This research work is therefore structured towards investigating the buying behavior of consumers towards Pepsi, Mirinda and 7up flavor with the aim of modeling their consumption behavior at proposed sales point. Findings in this work will be useful for businessmen, entrepreneurs in making prospective decision regarding the location of standard retail outlet based on the altitude of the populace towards beverages.

\subsection{Aim and Objectives}

This research work is designed to investigate the consumer buying behavior and the consistency of Dirichlet model in tracking consumers' habit in order to satisfy the following objectives:

(i) To predict the future behavior of consumer using Dirichlet model approach; 
(ii) To study the consistency of the model as a function of reliability on the estimate of the model as it affects buying behavior of consumers;

(iii) To suggest necessary recommendations; this would be useful in making quantitative decision and planning regarding the marketing of beverages.

\section{Research Methodology}

\subsection{Dirichlet model development}

The Dirichlet distribution is a multivariate generalization of the beta distribution. Recall that the beta distribution arose as follows; suppose that $x_{1}$ and $x_{2}$ are independent Gamma random variables with $x_{1} \sim G a\left(\alpha_{1}, \beta\right) \quad$ and $\quad x_{2} \sim_{G a\left(\alpha_{2}, \beta\right)}$ respectively. Then if $\mathrm{X}$ is defined by $X=\frac{x_{1}}{\left(x_{1}+x_{2}\right)}$, we have that $X \sim B e\left(\propto_{1}, \propto_{2}\right)$.Now consider a generalization; suppose that $x_{1}, x_{2}, x_{3} \ldots x_{k+1}$ are independent Gamma random variables with $x_{i} \sim{ }_{G a}\left(\propto_{i}, \beta\right), \quad$ fori $=1, \ldots, k+1$.

Define $X_{i}=\frac{X_{i}}{X_{1}+X_{2}+\cdots+X_{k+1}}$ fori $=1, \ldots, k+1$. Then the joint distribution of vector $X=\left(X_{1}, \ldots, X_{k}\right)^{T}$ is given by density

$f X_{1 \ldots,}, X_{k}\left(x_{1, \ldots,}, x_{k}\right)=\frac{\gamma(\alpha)}{\gamma_{\left(\alpha_{1}\right) \ldots \gamma\left(\alpha_{K}\right) r\left(\alpha_{K+1}\right)}} x_{1}^{\propto 1-1} \ldots x_{k}^{\propto k-1} x_{k+1}^{\propto k+1-1}$,

for $0 \leq x_{i} \leq 1$ for all isuch that $x_{i}+\cdots+x_{k}+x_{k+1}=1$, where $\propto=\alpha_{1}+\cdots+$ $\propto_{k+1}$ and where $x_{k+1}$ is defined by $x_{k+1}=1-\left(x_{1}+\cdots x_{k}\right)$. Equation (1) reduces to the beta distribution if $\mathrm{k}=1$.

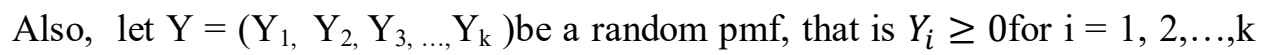
and $\sum_{i=1}^{k} Y_{i}=1$. In addition, suppose that $\alpha=\left(\alpha_{1}, \alpha_{2}, \ldots, \alpha_{k}\right)$ with $\alpha_{i}>0$ for each i, and let $\alpha_{0}=\sum_{i=1}^{k} \alpha_{i}$.Then, $\mathrm{Y}$ is said to have a Dirichlet distribution with parameter $\alpha$, which is denoted by $\mathrm{Y} \sim \operatorname{Dir}(\alpha)$, if it has $\mathrm{f}(\mathrm{y} ; \alpha)=0$ if $\mathrm{u}$ is not a pmf, and if $\mathrm{u}$ is a pmf then

$f(y ; \alpha)=\frac{\left(\alpha_{i}\right)}{\prod_{i=1}^{k+1}\left(\alpha_{i}\right)} \prod_{i=1}^{k+1} y_{i}^{\alpha_{i}-1}=m_{0}(x)=Y_{1}$

Then $f(y ; \alpha)$ can be re-written as $f(y ; \alpha)=\frac{1}{B(\alpha)} \prod_{i=1}^{k} x_{i}^{\alpha_{i}-1}$ where $\mathrm{B}(\alpha)$ $=\frac{\prod_{i=1}^{k} \Gamma\left(\alpha_{i}\right)}{\Gamma\left(\sum_{i=1}^{k} \alpha_{i}\right)}$ 


\subsubsection{Estimation of parameters}

The Dirichlet distribution is a model of how proportions vary. Let $\mathrm{p}$ denote a random vector whose elements sum to 1 , so that $p_{k}$ represents the proportion of item $k$. Under the Dirichlet model with parameter vector $\alpha$, the probability density at $\mathrm{p}$ is given by equation (3).

$$
\mathrm{P}(\mathrm{p}) \sim \mathrm{D}\left(\alpha_{1} \alpha_{2} \ldots \ldots . \alpha_{k}\right)=\frac{\left(\sum_{\mathrm{k}} \alpha_{\mathrm{k}}\right)}{\prod_{k} \sum\left(\alpha_{k}\right)} \prod_{k} P_{k}^{\alpha_{k}-1}, \text { where } p_{k}>0 \text { and } \sum_{k} p_{k=1}
$$

According to Minka (2000), the parameters can be estimated from a training set of proportions: $\mathrm{D}=\left(p_{1} \ldots \ldots . p_{N}\right)$. The maximum-likelihood estimate of $\alpha$ maximizes $\mathrm{p}(\mathrm{D} / \alpha)=\prod_{i} p\left(Y_{i} / \alpha\right)$. The log-likelihood can be written as:

$\log \mathrm{p}(\mathrm{D} / \alpha)=\mathrm{N} \log \left(\sum_{i} \alpha_{i}-N \sum_{i} \log \left(\alpha_{\mathrm{i}}\right)+\mathrm{N} \sum_{\mathrm{i}}\left(\alpha_{\mathrm{i}}-1\right)\right) \quad \log \bar{p}_{\imath}$

Where $=\log \bar{p}_{l} \quad=\frac{1}{N} \sum_{k} \log p_{k i}$

This objective is convex in $\alpha$ since Dirichlet is in the exponential family. This implies that the likelihood is unimodal and the maximum can be found by a simple search. A direct convexity proof has also been given by Ronning (1989). The gradient of the log-likelihood with respect to one $\alpha_{k}$ is given by (5).

$$
\begin{aligned}
& g_{k}=\frac{d \log (\mathrm{D} / \alpha)}{d \alpha_{k}}=N \Psi\left(\sum_{k} \alpha_{k}\right)-N \Psi\left(\alpha_{k}\right)+N \log \log \overline{p_{k}} \\
& \Psi(x)=\frac{d \log (\mathrm{x})}{d x}
\end{aligned}
$$

$\Psi$ is known as digamma function and almost similar to natural logarithm.

$$
\mathrm{E}\left(\log p_{k}\right)=\Psi\left(\alpha_{k}\right)-\Psi\left(\sum_{k} \alpha_{k}\right)
$$

In order to find the stationary point, we employed the Newton method of iteration. Now we obtain the derivative of equation (5) as defined in (7) and (8).

$$
\begin{aligned}
& g^{\prime \prime}{ }_{k}=\frac{d \log (\mathrm{D} / \alpha)}{d \alpha_{k}{ }^{2}}=N \Psi^{\prime}\left(\sum_{k} \alpha_{k}\right)-N \Psi^{\prime}\left(\alpha_{k}\right) \\
& \frac{d \log (\mathrm{D} / \alpha)}{d \alpha_{k} d \alpha_{j}}=N \Psi^{\prime}\left(\sum_{k} \alpha_{k}\right) \quad k \neq j
\end{aligned}
$$


$\Psi^{\prime \prime}$ is known as trigamma function. Then the estimate of the new parameter can be obtained by using (9) \& (10)

$$
\begin{gathered}
\alpha^{\text {new }}=\alpha^{\text {old }}-H_{g}{ }^{-1} \text { where }\left(H^{-1}\right)_{k}=\frac{g_{k-b}}{q_{k k}} \\
\mathrm{~b}=\frac{\sum_{j} q_{j j / q_{j j}}}{\frac{1}{z}+\sum_{j} \frac{1}{q_{j j}}}, \quad \mathrm{Z}=\mathrm{N} \Psi^{\prime}\left(\sum_{k} \alpha_{k}\right) \text { and } q_{j j}=\mathrm{N} \Psi^{\prime}\left(\alpha_{k}\right) \delta(j-k)
\end{gathered}
$$

$\alpha^{\text {old }}$ is the vector of initial guess so that the new parameters will be estimated via iterative Newton Raphson algorithm. The initial guess was chosen and simple R-code was written which was implemented by R-programming language to obtain the parameters estimates (parameter vector) for the Dirichlet model so that the estimate will be used to obtain stochastic matrix, Bayes factor and the behavior of consumer at different proposed sales point with a view to accurately model the buying habit of consumers through Dirichlet model.

In this research work, we defined the variance-covariance to be $\sum$, Stochastic matrix to be $\mathrm{P}$ and correlation matrix to be $\rho$.

2.1.2Stochastic matrix: let $\mathrm{P}$ represented by $\left[P_{i j}\right]$ be a $\mathrm{n} \times \mathrm{n}$ square matrix, then $\mathrm{P}$ is known as a stochastic matrix if the following conditions are satisfied.

(i) All the element of $\mathrm{P}$ are non-negative numbers, that is, for each $i, P_{i j} \geq 0$.

(ii) $\quad \sum_{i=1}^{n} P_{i j}=1$

In this context, the columns of matrix $P$ are labeled: Pepsi, 7up and Mirinda flavor so that each probability value corresponding to any of these flavors in the matrix is the proportion of consumers' behavior towards such flavors within the period of investigation.

2.1.3Variance-covariance matrix: the covariance matrix or variancecovariance matrix $\sum$ is the matrix whose entry $(\mathrm{i}, \mathrm{j})$ is the covariance between $i$ and $j$.

It can be represented mathematically as defined in (11).

$\sum_{i j}=\operatorname{cov}\left(X_{i}, X_{j}\right), \sum^{-1}$ exist

The rows and columns of matrix $\sum$ are both labeled: Pepsi, 7up and Mirinda which mean the element of $\sum$ at exactly $\sum_{i j}$ (where $i=$ pepsi and $j=7 u p$ ) is the covariance between Pepsi and 7 up flavor. 
2.1.4Correlation matrix: this matrix is closely related to variance-covariance matrix and is the matrix of Pearson product moment correlation coefficient between each of the variables (Flavors) in a random vector $\mathrm{X}$ which can be written as (12).

$$
\rho=D^{-1} \sum D^{-1}
$$

Where $\sum=$ covariance matrix and $\mathrm{D}$ is the matrix of the diagonal elements of $\sum$ and $D^{-1}$ is its inverse. Each element on the principal diagonal of $\rho$ is the correlation of a random variable (such as Pepsi, 7up or Mirinda) with itself which always equals 1 . The rows and columns of matrix $\rho$ are both labeled: Pepsi, 7up and Mirinda which mean the element of $\rho$ at exactly $\rho_{i j}$ (where $i=$ pepsi and $j=7 u p$ ) is the correlation between pepsi and 7 up flavor. $\rho_{i j}$ Is the correlation between Pepsi and Mirinda, if $i=$ pepsi and $j=$ mirinda.

\subsection{Source of Data and Scope Coverage}

The result reported in this research work is based purely on secondary data available from wholesales standard outlets of beverages outfit for period of eight consecutive years (2008 to 2016). These reports have been generated with the help of sales record (purchase data), precisely 7up Bottling Company Nig. Plc (Ilorin Depot), available in the outlet which showcases the identity of customers as they patronize the outlets and eventually buy the products.

\section{Results and Discussion}

\subsection{Fitting Dirichlet Distribution to the Buying Behavior Data}

Table1: Parameter Estimate of Dirichlet Distribution

\begin{tabular}{lllll}
\hline coefficients & Estimate & St. Error & Z-value & $\operatorname{Pr}(>|\mathrm{Z}|)$ \\
\hline$\alpha_{1}$ & 4.145 & 0.354 & 11.71 & $<2 \mathrm{e}-16^{*}$ \\
$\alpha_{2}$ & 4.375 & 0.354 & 12.36 & $<2 \mathrm{e}-16^{*}$ \\
$\alpha_{3}$ & 4.790 & 0.352 & 13.36 & $<2 \mathrm{e}-16^{* *}$ \\
\hline
\end{tabular}

Table 1 above shows the parameter vector to be $\alpha=\left(\begin{array}{lll}4.145 & 4.375 & 4.790\end{array}\right)$, the statistical significance test shows that all the parameters are statistically significant, this is because $\mathrm{P}_{\text {-value }}(2 \mathrm{e}-16)<\alpha(0.05)$. This determines significantly 
the shape of the Dirichlet distribution and how important are those estimates in making decision as regards the future sales volume of Pepsi, 7up and Mirinda. Mirinda flavor is statistically significant as compared with other flavors under investigation

\subsection{Parameter Vector and Probability Vector}

The parameters of the Dirichlet distribution is represented by the vector $\alpha_{\mathrm{i}}=$ $\left(\begin{array}{lll}4.145 & 4.375 & 4.790\end{array}\right)$ with corresponding probability vector $\mathrm{m}_{\mathrm{i}}=(0.2400182$ 0.30224450 .45777375 ), the vector $m_{i}$ is called normalized parameters vector. From the parameter vector, we can infer that the purchase rate for Pepsi, 7 up and Mirinda vary almost uniformly. The flavors under investigation contribute significantly to the sales volume of 7 up Bottling Company Nig. Plc with Mirinda contributing the most. This shows that there will be an increment of over $45 \%$ in purchase of Mirinda and over 30\% increment will be expected in 7 up while Pepsi shows increment in purchase for over $20 \%$ for the periods under review. The normalized parameter vector described the rate of purchase of each of the flavor under investigation, and it shows that Mirinda has the highest purchase rate.

\subsection{Precision}

Since the precision parameter $S(0.04301668)<1$, then the model is consistent for buying behavior data. It does not exaggerate the parameter vector $\alpha=\left(\alpha_{i}\right)$ and this constitute to the high consistency and efficiency of the model .When the Bayes factor $(\mathrm{BF})(1.181855 \mathrm{e}-6)<1$, is less than one, the fit is also good for Dirichlet model.

\subsection{Stochastic Matrix}

$$
\mathbf{P}=\left[\begin{array}{lll}
0.2400182 & 0.3022445 & 0.45777375 \\
0.2400182 & 0.3022445 & 0.45777375 \\
0.2400182 & 0.3022445 & 0.45777375 \\
0.2400182 & 0.3022445 & 0.45777375 \\
0.2400182 & 0.3022445 & 0.45777375 \\
0.2400182 & 0.3022445 & 0.45777375
\end{array}\right]
$$

From the matrix $\mathrm{P}$ above, we can deduce that the rows of the matrix are all probability vector and it describes the purchasing habit of consumer regarding Pepsi, 7up and Mirinda throughout the periods under review. From P, we can 
infer that throughout the periods, the purchase proportion for Mirinda, 7up and Pepsi are $45.7774 \%, 30.2245 \%$ and $24.0018 \%$ respectively. As we can see that the purchase rate and purchase proportion are constant.

\subsection{The probability of Sales in the Next Eight Sale Period}

Table 2: Predictive probabilities

\begin{tabular}{llll}
\hline Sale Periods & Pepsi & 7up & Mirinda \\
\hline 1 & 0.119091 & 0.011019 & 0.809890 \\
2 & 0.100215 & 0.782130 & 0.530260 \\
3 & 0.726510 & 0.108880 & 0.830890 \\
4 & 0.131521 & 0.133232 & 0.1335251 \\
5 & 0.742120 & 0.761120 & 0.580410 \\
6 & 0.900520 & 0.622140 & 0.540860 \\
7 & 0.104231 & 0.902620 & 0.980450 \\
8 & 0.132115 & 0.125901 & 0.149534 \\
\hline
\end{tabular}

From Table 2 above, we can deduce that the sales pattern is stochastic for the proposed eight sales periods, as no flavor dominated in having significantly higher sales throughout the period. Mirinda Flavor will have significantly higher sales as compared to others with average purchasing probability $56.9478 \%$ within the periods. 7up will possess higher sales in sales period two as compared to others with probability of sale 0.782130 . Pepsi and Mirinda will dominate the 7 up at sales period three with their corresponding purchasing probabilities 0.726510 and 0.830890 ( $72.651 \%$ and $83.089 \%)$, respectively. At the fourth sales period, there will be low sale of all the flavors under investigation due to the evidence obtained from their probability of sales as predicted for those periods which are $0.131521,0.133232$ and 0.1335251 for Pepsi, 7up and Miranda, respectively. At period five, the probability of sales will be 0.742120 , 0.761120 , and 0.580410 for Pepsi, 7up and Mirinda: showing that Mirinda will have lower sales as compared to Pepsi and 7up at that period. At sixth period, Pepsi will have the highest sales with probability of about 0.900520 . About 
$0.622140(62.214 \%)$ and $0.540860(54.0860 \%)$ sales will be expected for 7up and Mirinda, respectively. Although they will all experience higher sales at this period, but Pepsi will have more significantly higher sales at period sixth. At seventh period, Mirinda and 7up will both have approximately and significantly higher sale with probability of about 0.980450 and 0.902620 respectively as compared to Pepsi with probability 0.104231 . There won't be more sales for all the products at the eighth period. Throughout the whole proposed sale period the average probability of sales are $0.368518,0.361292$ and 0.569478 for Pepsi, 7 up and Mirinda respectively. This shows that the Dirichlet model is said to be more efficient for the purchase of Miranda, because the data fits reasonably well. Therefore, Mirinda is said to have more sales throughout the proposed sales periods with average sales of over $56 \%$. That is, over $56 \%$ of the expected sales for the proposed sales period will be experienced by Mirinda flavor.

The eight periods is hypothetically any eight prospective sales periods. The next eight consecutive expected sales. Those eight periods could be within a week or in just two days. The volume of products or flavours available for sales at any periods will determine when those eight consecutive periods will be. Absolute Probability Distribution was used to determine the probability of sale of each flavour at these different periods, when we assume that sales pattern follow random walk (which is a special form of stochastic process).

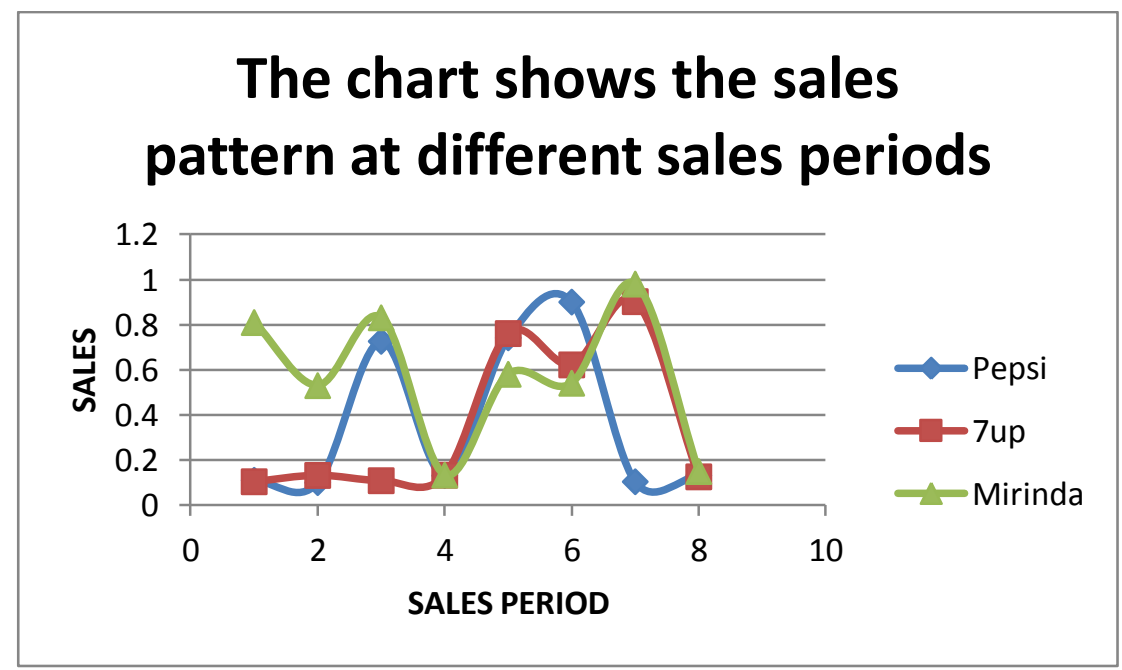

Figure 1: plot which shows the probability of sales at different proposed sales periods 


\subsection{Discussion}

The research work has modeled the buying patterns of Pepsi,7up and Mirinda, and there is an increase towards purchasing more of Mirinda as compared to other flavors with probability of purchase of over $56 \%$. In fact, the percentage of Mirinda which will be purchased in the future is significantly higher with about 72.651\%in three consecutive sales periods, as compared to 7up and Pepsi with over $30.067 \%$ and $31.527 \%$ respectively. According to early economists, Adam Smith (1776) and Alfred Marshall (1890): economic theory holds that purchasing decisions are the result of largely "rational" and conscious economic calculations. Thus, the individual buyer seeks to spend his income on those goods that will deliver the most utility (satisfaction) according to his tastes and relative prices.

\subsection{Conclusions}

The Dirichlet model provides a good basis for predicted values regarding the three products under investigation. The Dirichlet can therefore be relied on for accurate, adequate and consistent decision making and planning.

\subsection{Recommendation}

(i) The management should ensure they take adequate control measures so that the great decline in the sales volume for 7up at the next eighth period will be corrected in order to find a way to proactively divert the company from loss. By taking proactive steps towards the forecasted periods where there will be great reduction in sales of all the major flavors, the company may in turn experience sales boom at that sales period.

(ii) They should also provide adequate and tenable information that would be of great help to the prospective customers or buyers so that their expected sales would be realized as being stated by Dirichlet Model. 


\section{References}

1. Andrew S.C., Mark D.U., Gerald J. G.,(2004). Understanding brand Performance Measure using Dirichlet Benchmarks, Journal of Business research 57

1307-1325. https://doi.org/10.1016/j.jbusres.2002.11.001

2. Ehrenberg ASC.,(1959).The pattern of consumer purchases. Appl Stat 1959;8:26-41. https://doi.org/10.2307/2985810

3. Ehrenberg ASC, Goodhardt GJ.,(2000) New brands: near-instant loyalty. J MarkManage 2000;16(6):607-17 [Revised version] https://doi.org/10.1362/026725700785045912

4. Fader, P. S. and D. C. Schmittlein. (1993). Excess behavioral loyalty for high-share brands: Deviations from the Dirichlet model for repeat purchasing. J. Marketing Res. 30(4), 478493https://doi.org/10.2307/3172692

5. Marie, C.G., Radka, S., Mathew, P.C., Pauline, M.R., Thomas, B.M., (2013). Greedy feature selection for glycan chromatography data with generalized dirichletdistribuition, BMC Bioinformatic14:15 https://doi.org/10.1186/1471-2105-14-155

6. MacKay, D. J. C. (1998). Choice of basis for Laplace approximation. Machine Learning, 33. http://wol.ra.phy.cam.ac.uk/mackay/abstracts/laplace.html. https://doi.org/10.1023/A:1007558615313

7. Thomas M.P.,(2000)Estimating a Dirichlet distribution. research.microsoft.com/ minka/papers/dirichlet/, 2000.

8. Utkin, L.V.,(2005). Extensions of belief functions and possibility distributions by using the impreciseDirichlet model. Fuzzy Sets and Systems,

154:413-431. https://doi.org/10.1016/j.fss.2005.03.012

9. Uncles MD, Ehrenberg ASC, Hammond K(1995). Patterns of buyer behavior:regularities, models and extensions. Mark Sci; 14(3):G71 - 8 . https://doi.org/10.1287/mksc.14.3.G71

10. Uncles M.D., Dowling G., Hammond K.(2003). Customer loyalty and customer loyalty programs. J Consum Mark; 20(4):294- 316 https://doi.org/10.1108/07363760310483676 


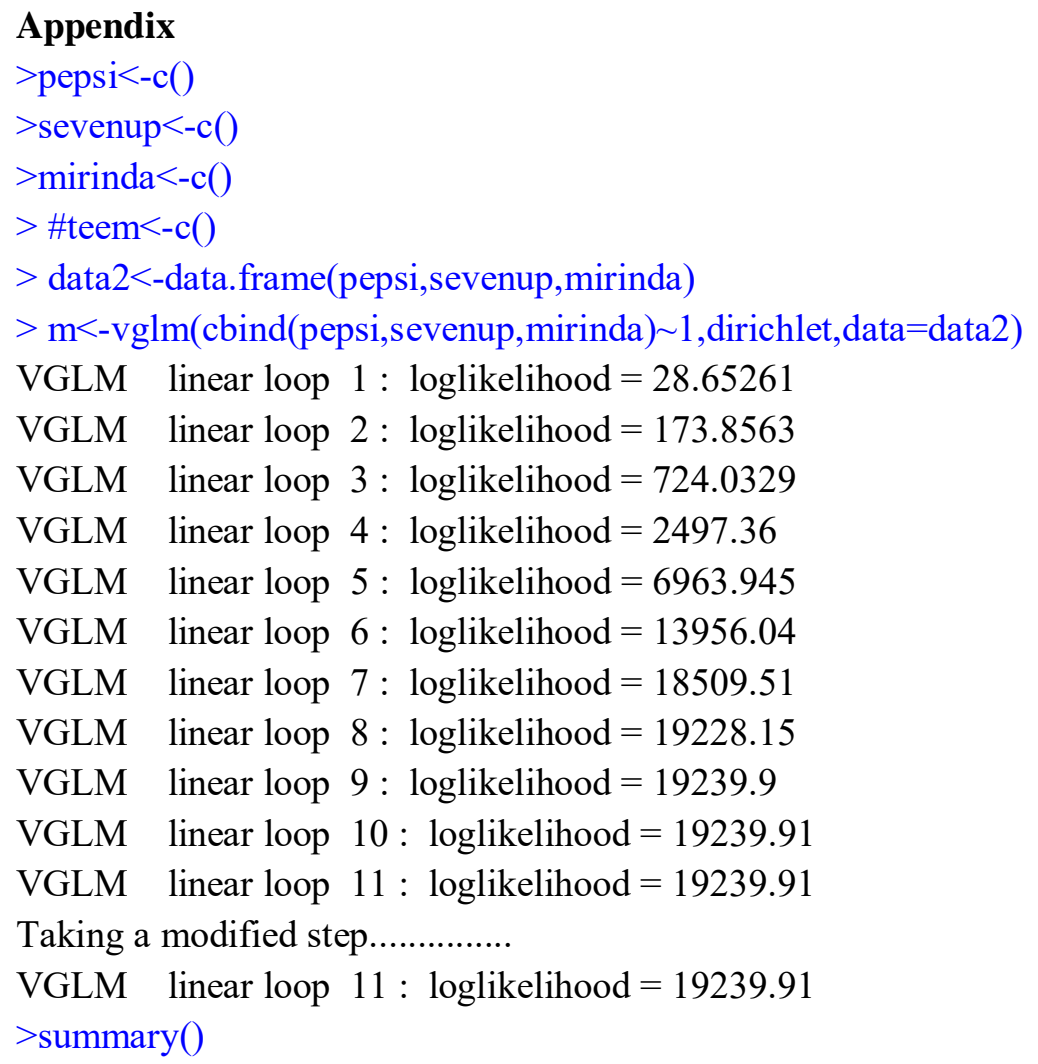

Call:

$\operatorname{vglm}($ formula $=\operatorname{cbind}($ pepsi, sevenup, mirinda $) \sim 1$, family $=$ dirichlet, data $=$ data 2 , trace $=$ TRUE)

Pearson residuals:

Min 1Q Median 3Q Max

loge(shape1) -148.5 -58.98 28.07 70.62101 .69

loge(shape2) -152.4 -62.78 27.0882 .53101 .78

loge(shape3) -162.3 -53.20 31.84 81.3797 .41

Coefficients:

Estimate Std. Error $\mathrm{z}$ value $\operatorname{Pr}(>|z|)$

$\begin{array}{lllll}\text { (Intercept):1 } & 4.145 & 0.354 & 11.71 & <2 \mathrm{e}-16^{* *} \\ \text { (Intercept):2 } & 4.375 & 0.354 & 12.36 & <2 \mathrm{e}-16^{* *} \\ \text { (Intercept):3 } & 4.790 & 0.354 & 13.53 & <2 \mathrm{e}-16^{* * *}\end{array}$


Signif. codes: 0 ‘***’ 0.001 '**’ 0.01 '*’ 0.05 ‘' 0.1 ' 1

Number of linear predictors: 3

Names of linear predictors: loge(shape1), loge(shape2), loge(shape3)

Dispersion Parameter for dirichlet family: 1

Log-likelihood: 19239.91 on 21 degrees of freedom

Number of iterations: 11

$>\operatorname{coef}()$

(Intercept):1 (Intercept):2 (Intercept):3
4.144883
4.375405
4.790464

$>\operatorname{coef}(\mathrm{m}$, matrix $=$ TRUE)

loge(shape1) loge(shape2) loge(shape3)

$\begin{array}{llll}\text { (Intercept) } & 4.144883 & 4.375405 & 4.790464\end{array}$

$>\operatorname{vcov}()$

$>$

(Intercept):1 (Intercept):2 (Intercept):3

(Intercept): 1

0.1253384

0.1235753

0.1238401

(Intercept):2

0.1235753

0.1253405

0.1240427

(Intercept):3

0.1238401

0.1240427

0.1253429

$>\mathrm{x}<-\operatorname{cov} 2 \operatorname{cor}(\mathrm{p})$

$>\mathrm{x}$

(Intercept):1 (Intercept):2 (Intercept):3

(Intercept): $1 \quad 1.0000000 \quad 0.9859248 \quad 0.9880285$

(Intercept):2 $\quad 0.9859248 \quad 1.0000000 \quad 0.9896370$

(Intercept): $3 \quad 0.9880285 \quad 0.9896370 \quad 1.0000000$

$>$ depar(m)

$>$ head(fitted(m))

pepsisevenupmirinda

10.24001820 .30224450 .4577374

20.24001820 .30224450 .4577374

30.24001820 .30224450 .4577374

40.24001820 .30224450 .4577374

pepsisevenupmirinda 
Dirichlet Model: Its Consistency and Tracking Pattern Using Buying Behavior Data

10.1190910 .1101190 .809890

20.1002150 .7821300 .530260

30.7265100 .1088800 .830890

40.1315210 .1332320 .115251

50.7421200 .7611200 .580410

60.9005200 .6221400 .540860

70.1042310 .9026200 .980450

80.1321150 .1259010 .149534

\#bayes factor

$>$ b $<-((\operatorname{gamma}(4.145) * \operatorname{gamma}(4.375) * \operatorname{gamma}(4.790) / \operatorname{gamma}(\mathrm{s})))$

$>\mathrm{b}$

[1] $1.181855 \mathrm{e}-06$ 